\title{
Application Research of Human Resources Management Based on Safety Occupational Aptitude
}

\author{
Bin ZHENG ${ }^{1, a}$, Sheng-Sheng WU ${ }^{2, b,{ }^{*}}$ \\ ${ }^{1}$ Jiao Da East Road Jiashu Qu, Tower \#5,1408, Beijing Jiaotong University,Beijing Haidian District, \\ P.R. of China \\ ${ }^{2}$ The $6^{\text {th }}-9$, north Avenue Fuchengmen, Xicheng District, Beijing, P.R. of China \\ aZhengb16@263.net, 'bsscello@hotmail.com \\ ${ }^{*}$ Corresponding author
}

Keywords: Safety occupational aptitude, Competency model, Human resources.

\begin{abstract}
By reviewing the relevant theories, the definition and research object of safety occupational aptitude research has been cleared. By introducing the competency theory in human resource management into the research of safety occupational aptitude and drawing reference from the establishment and inspection method of competency model, this paper provides a new method and theoretical basis for establishing special industry safety competency model.
\end{abstract}

\section{Introduction}

Industrial safety is the first priority in social and economic activities. By analyzing the accident-causing theory, it is revealed that human error is one of the major reasons causing accidents. Statistics shows that more than $80 \%$ of the accidents are caused by human error. Therefore, human factor is the critical reason to the accidents.

Individual difference theory tells us that every individual has something different form each other. Research on safety occupational aptitude and human psychology can help to more efficiently utilize human resource and carry out psychology training in order to avoid accident (Song 2003). Scientific and accurate safety evaluation can be reached by considering human behavior, physical and psychological conditions (Ye 2003). Therefore, in a man-machine system, by applying the research methods adopted in psychology and behavioral studies to evaluate safety occupational aptitude can effectively avoid accidents.

\section{Background and theory review}

\section{Domestic and international research on Occupational Aptitude Research}

Occupational aptitude research mainly focuses on two aspects in developed countries. One is on jobs requiring special skills such as pilots, train divers and sailors while the other is on normal jobs and the general public, with research on career development, adaptability when changing job and so on. The occupational aptitude research has extended the career consultation fields.

With the development of transportation industry, electricity industry and manufacturing industry, more attention has been paid on human factors. Occupational aptitude has reached a great progress. Research on individuals who are engaged in operation of dangerous devises provides 27 physical or psychological benchmarks in occupational activities (Wu \& Zhang 1997). Following this research trend, 13 benchmarks for crane worker (Jin \& Dai 1997) and 6 selective benchmarks for electrical operator were made (Ye \& Sheng 2003).

\section{Concept and Object of Safety Occupational Aptitude}

Safety Occupational Aptitude is based on the human factor engineering theory which talks about how human fit the machine. Safety Occupational Aptitude is the application of research on safety psychology and behavior (Li 2009). From this aspect, Safety Occupational Aptitude is defined as 
the man-machine adaptability and physical and psychological consciousness of safety that an individual has when carrying out work which are essential to self-protection and self-adjustment (Li \& Song 2009).

Competence is a standardized requirement for an individual to properly perform a special job. It is a combination of knowledge, skills and behavior which are utilized to improve performance. More generally, competence is the state or quality of being adequately or well qualified to perform a specific role. Hence, Safety Occupational Aptitude's research object is the individuals in the special industry. The research pinpoints the individual safety capacity, combined with recognition competence, physical and psychological quality and practical operation capacity.

\section{Competency model}

In 1973, Dr. McClelland, a professor in Harvard Business School of Psychology, published his article, Testing for Competency rather than Intelligence. He illustrated that competency is the special characteristic that an individual has who always has outstanding performance in his work. A competency model refers to a group of competencies, which usually range between 7 to 9 different kind of competencies that are required in a particular job. The number and type of competencies in a model will depend upon the nature and complexity of work and the culture and values of the organization in which the work takes place. In his article, Dr. McClelland also found that traditional tests such as academic aptitude and knowledge tests did not predict success in the job. Since then, many leading organizations have started using competency as a benchmark in recruiting, selecting and managing their employees' outstanding performers.

One of the most famous competency models is the Iceberg Model (Table 1 lists 6 elements).

Table 1 List of the Iceberg model Elements

\begin{tabular}{|c|c|}
\hline Elements & Operational Definition \\
\hline Skills & Things an individual can do well \\
\hline Knowledge & A specific topic that an individual knows \\
\hline Social role & $\begin{array}{l}\text { An image that an individual displays in public. It reflects the values of } \\
\text { that individual }\end{array}$ \\
\hline Self-image & The view an individual has about himself. It reflects his self-identity. \\
\hline Traits & $\begin{array}{l}\text { The enduring characteristics of an individual. They reflect the way the } \\
\text { individual tends to describe people. }\end{array}$ \\
\hline Motives & Unconscious thoughts and preferences, which drive behavior. \\
\hline
\end{tabular}

\section{Construction of safety occupational aptitude model}

There are different ways used by domestic researchers to extract human benchmarks. This paper is trying to bring competency model of human recourse management into safety occupational aptitude in order to provide a new theory.

Spencer modified Dr. McClelland's competency model and came up with the Spencer model which was more systematic and accurate and especially targeted the working environment and job's potion characteristics. We can also use the method for reference.

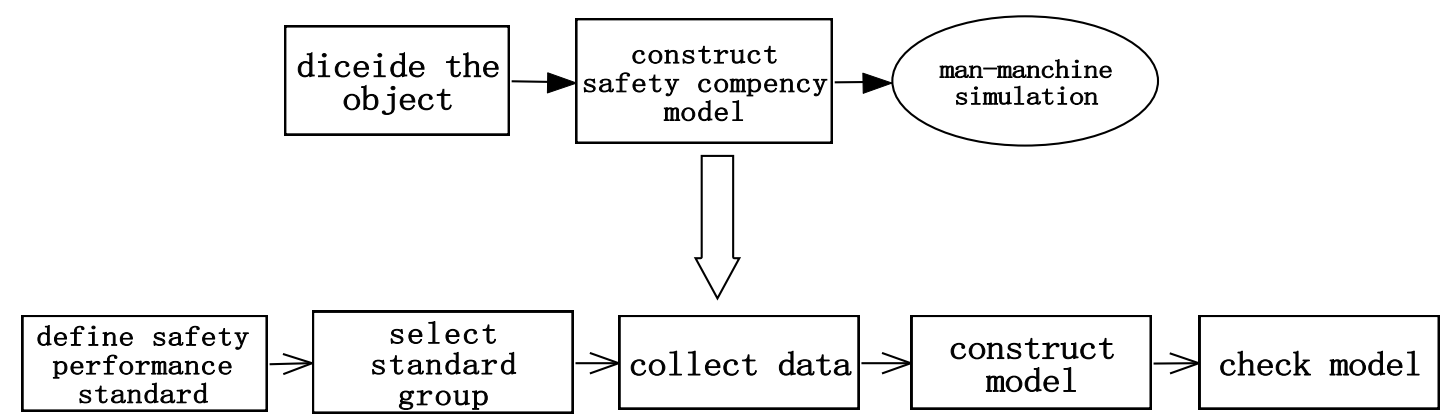

Fig. 1 human safety occupation aptitude model construction process. 


\section{Decide safety evaluation object}

At the beginning of the model constructing, the first thing is to decide the safety evaluation object. Safety occupation aptitude is based on the evaluation of individual's safety competency and working adaptability. Therefore the model needs to consider the job level, organization environment, safety motivation, safety management and etc.

\section{Define safety performance standard}

Establishing the performance standard at the outset of the model is also critical because different working industries have different standards. However, the general standard is doing the job efficiently and safely. Normally, job description, occupational standards, job skills and safety recognition are widely used in this stage.

\section{Select standard group}

It is normal to establish a standard group to manage the model, and the group members should agree that establishing such a model is for the better performance in a job. The criteria should include hard data if possible (e.g., productivity figures), but other criteria such as managerial effectiveness as measured by direct reports or peer ratings may also be included. Avoid using a single measure.

The standard group sample consists of individuals from two distinct groups - a safe and unsafe performer - to understand what really differentiates performance and creates safety in a job. It is important to consider three factors when selecting people for the group. The first factor is that the outstanding safety performers should be the absolute best - those who are rated high on all or most of the performance criteria. The second factor is that there needs to be a control or contrast group of fully acceptable performers. Lastly the groups should be large enough to allow for statistical analysis.

From the experience, a total group that consists of $10 \%$ of the position of interest within organization should allow for proper statistical analysis. However, if the total population is small, a higher proportion will be needed. As a rule of thumb, it is advantageous to weigh groups more heavily towards superior performers - there is always a lot to learn from the best safe performers.

It is important to note that if the safety occupational aptitude model is being developed to change culture or aligning people behind a new strategy, then it might not be desirable to select those who were top safe performers under the old culture or strategy. Rather, a group that is already executing the new strategy or acting in accordance with the new culture would be selected as a comparator group. If there are no "hard" measures, then a nomination approach can be employed by having direct reports, peers, and coaches' vote.

\section{Collect data}

Data may be collected through Behavioral Event Interviews (BEIs) or other assessment methods. The most common ones are expert panels or focus groups.

BEI. The BEI is the most effective assessment method. It is a structured interview similar to a critical incident interview, but focused on the individual and their competencies rather than the tasks. It is a process for indirectly collecting samples of behavior, which have led to success in the role the person has, as well as events where he or she has been frustrated in delivering what they wanted to do.

The interview requires a high-degree of rigor from the interviewer and-if done well-will reveal patterns of intentional behavior that deliver outstanding performance. The interview focuses on thoughts, feelings, behaviors, and outcomes. A comparison of all outstanding performers yields an excellent view of how they deliver against the performance criteria and, when contrasted with average performers, results in a template of excellent performance in that particular role or job and which is specific to the organization. It is very important that BEI be a "double blind" process to remove any bias. That is, neither the interviewers nor the interviewees know if they are in the 
"outstanding" or the "more typical" samples.

The results of a BEI not only provide the competencies, but also give rich data about the context in which the competencies are displayed and how they operate together. This can reveal valuable information that can be used to help others develop competencies. BEIs give the best results, and for key jobs or complex roles they are well worth the investment.

Expert Panels. Expert panels, or focus groups, use people who are knowledgeable about the job, such as outstanding performers, customers, HR specialists, and line managers. The panel brainstorms the competencies needed to achieve outstanding performance in the job. Expert support is required to prevent the panel from producing either over-generalized competencies or is overly influenced by any one group. Panels typically do not generate the full range of competencies. In fact, they often miss some the most important, deeply rooted competencies. From our experience, panel data is only about $50 \%$ as accurate as BEI data.

Surveys. Another method of collecting data is through surveys. While this is a quick way of generating data from a broad population, it is limited because it only provides responses to the questions asked. And, like expert panels, surveys often miss the hidden competencies that are unique to the job or the organization. Augmenting surveys with BEIs can improve the outcome, but still will not provide the richness of a model created from a larger number of BEIs.

\section{Construct model}

Constructing the models from data is the most complex part of the process. The data need to be analyzed thematically, i.e., in clusters according to themes related to patterns that are discernable in the interview. These will then generate hypotheses on what the competencies are and how they work together to produce outstanding performance.

The desired outcome is a comparison of what outstanding performers do differently from average performers. If the two groups have been chosen well, the differences often stand out very clearly. The data are usually analyzed both thematically from the interviews and statistically to test for real differences between the two groups.

The output may take the form of a "safety competency dictionary" that includes all the competencies, their definitions, and descriptions of the levels expressed as interval scales together with behavioral examples of the competency. The format depends on the nature of the applications for which the model will be used.

Based on competency job potion analyze and document study, Wang \& Chen (2002) used structure interviewing and rating scale to create the top manager competency. Zhong \& Shi (2004) used BEIs and average and star group study to create family enterprise top manager competency. Cheng \& Lu (2010) used document study, network information analyze, and expert panels to create college senior researchers competency benchmarks.

\section{Check model}

There are several ways to validate the model. Where there are large numbers of job-holders, it may be possible to create questionnaires based on the model and to administer them to a wider sample of jobholders. This includes both safe and unsafe performers. The analysis of this data will test the model and allow revisions and refinements to take place.

The constructed model can be used in human recourse practice, like recruit tests, 360 degree feedback or training programs. The model can be validated by observing the safe and unsafe performers before and after training.

Researchers usually tend to use the rating scale. When the survey data had been collected, researchers use cross validation method, criterion related validity and nomological validity etc. to check the model.

Cross validation, sometimes called rotation estimation, is a technique for assessing how the results of a statistical analysis will generalize to an independent data set. It is mainly used in settings where the goal is prediction, and one wants to estimate how accurately a predictive model will perform in practice. One round of cross validation involves partitioning a sample of data into 
complementary subsets, performing the analysis on one subset (called the training set), and validating the analysis on the other subset (called the validation set or testing set). To reduce variability, multiple rounds of cross-validation are performed using different partitions, and the validation results are averaged over the rounds. Wang (2002) used structural equation to create the senior manager competency model and used cross validation checked the model.

Criterion related validity is a measure of how well one variable or set of variables predicts an outcome based on information from other variables, and will be achieved if a set of measures from a personality test relate to a behavioral criterion. A typical way to achieve this is in relation to the extent to which a score on a personality test can predict future performance or behavior. Another way involves correlating test scores with another established test that also measures the same personality characteristic.

Nomological validity is a form of construct validity. It is the degree to which a construct behaves as it should within a system of related constructs be called a nomological set. Li (2006) created performance variable and used nomological validity to check the public official competency model.

Data from the selection processes or performance reviews should be kept to validate and update the model in the future in case there are insufficient numbers for significant validation to occur. This data collection is important because jobs evolve as situations change, and the competency framework will also have to be adjusted to reflect the new requirements. Often the competencies themselves do not change because the underlying motivational aspects of the jobs and job holders remain constant. However, the way in which the behaviors reflect the underlying drivers may change.

\section{Conclusion}

So, why are some people safer than the others? The answer is partly a function of the individual and partly a function of how they fit in the man-machine system in a particular role.

The current researches on creating a model of safety occupational aptitude are relatively less; most of the researches are in the benchmark-level. And the methods to extract benchmarks are different between researchers.

This paper provides the theoretical foundation and a new method to evaluate human safety factor by making reference to and developing the competency theory and model. This method can be used in recruiting new workers, reducing and preventing the accident happening and increasing the level of safety management. Safety occupational aptitude is essential to outstanding safety performance in a job which an individual work must equip or can be equipped through proper training. However, further research is still needed in relation to the benchmark measurement and evaluation and the detailed training program.

When organizations make the effort to apply safety occupational aptitude to select and develop individuals for safety-production, they avoid the collateral damage-recruiting costs, low morale, dissatisfied customers, missed opportunities - associated with a bad hire. And, most importantly, they greatly increase the odds that selected individual safe perform at a very high level and help drive the industry works safer.

\section{References}

[1] McClelland D C. Testing for Competence Rather Than for Intelligence [J]. American Psychologist, 1973, $28: 1-14$.

[2] Spencer L M, Spencer S M. Competence At Work: Model for Superior Performance. New York: John Wi2ley \& Sons. 1993.

[3] Hannaman G W, Spurgin A J, Lukic Y, Human Cognitive Reliability Model for PRA Analysis.NUS-4531, 1984. 
[4] Swain A D. Accident Sequence Evaluation Program Human Reliability Analysis Procedure [M] NUREG/CR-4772, 1987.

[5] Samanta P K, Brien J N O', Morrison H W. Multiple Sequential Failure Model: Evaluation of and Procedure for Human Error Dependency. [M]NUREG/CR-3837, Washington, DC (USA), 1985.

[6] Williams J C. A Data-Based Method for Assessing and Reducing Human Error to Improve Operational Performance. Proceedings of the IEEE 4th Conference on Human Factor in Power Plants, Monterey, California. Institute of Electronic and Electrical Engineers, New York (USA), 1988. 